\title{
LANGLANDS PARAMETERS ASSOCIATED TO SPECIAL MAXIMAL PARAHORIC SPHERICAL REPRESENTATIONS
}

\author{
MANISH MISHRA \\ (Communicated by Pham Huu Tiep)
}

\begin{abstract}
We describe the image, under the local Langlands correspondence for tori, of the characters of a torus which are trivial on its Iwahori subgroup. Let $k$ be a non-archimedian local field. Let $\boldsymbol{G}$ be a connected reductive group defined over $k$, which is quasi-split and split over a tamely ramified extension. Let $K$ be a special maximal parahoric subgroup of $\boldsymbol{G}(k)$. To the class of representations of $\boldsymbol{G}(k)$ having a non-zero vector fixed under $K$, we establish a bijection, in a natural way, with the twisted semisimple conjugacy classes of the inertia fixed subgroup of the dual group $\hat{\boldsymbol{G}}$. These results generalize the well known classical results to the ramified case.
\end{abstract}

\section{INTRODUCTION}

Let $k$ be a non-archimedian local field. Let $\boldsymbol{G}$ be an unramified connected reductive group defined over $k$ and let $\pi$ be a smooth, irreducible, admissible representation of $\boldsymbol{G}$ which is unramified; i.e., there is a hyperspecial subgroup $K$ of $\boldsymbol{G}(k)$ such that the $K$-invariant subspace $\pi^{K}$ of the space realizing $\pi$ is non-zero. One can associate a Langlands parameter to the representation $\pi$ via the following recipe.

The representation $\pi$ corresponds, in a natural way, to a character of the Hecke algebra $\mathscr{H}(\boldsymbol{G}(k), K)$. Then via the Satake isomorphism, this character corresponds to an unramified character $\chi$ of $\boldsymbol{T}(k)$ for certain maximal torus $\boldsymbol{T}$. This character $\chi$ is unique up to the relative Weyl group conjugacy. The character $\chi$, under the local Langlands correspondence for tori, corresponds to a Langlands parameter $\varphi_{\chi} \in$ $\mathrm{H}^{1}\left(W_{k}, \hat{\boldsymbol{T}}\right)$, where $\hat{\boldsymbol{T}}$ is the dual torus and $W_{k}$ is the Weil group. This parameter is induced from a cocycle in $\mathrm{H}^{1}\left(W_{k} / I_{k}, \hat{\boldsymbol{T}}\right)$, where $I_{k}$ is the inertia subgroup of $W_{k}$. Using this, one can then associate to the parameter $\varphi_{\chi}$ a semisimple conjugacy class in $\hat{\boldsymbol{G}} \rtimes\left(W_{k} / I_{k}\right)$, where $\hat{\boldsymbol{G}}$ is the complex dual of $\boldsymbol{G}$. This semisimple conjugacy class describes the Langlands parameter associated to $\pi$.

All these classic results are well known and can be found in [2]. We wish to find analogous statements when $\boldsymbol{G}$ is not necessarily unramified. Let $\boldsymbol{G}$ be quasi-split and tamely ramified. Let $K$ be a special maximal parahoric subgroup of $\boldsymbol{G}$ and let $\pi$ be a smooth, irreducible, admissible representation of $\boldsymbol{G}$ which is $K$-spherical, i.e., $\pi^{K} \neq 0$. We associate to $\pi$ a character $\chi$ of $\boldsymbol{T}(k)$ of a certain maximal torus $\boldsymbol{T}$ in a similar way as above, using the description of special maximal parahoric Hecke algebras given in [4. We show that $\chi$ is trivial on the Iwahori subgroup

\footnotetext{
Received by the editors April 20, 2013 and, in revised form, October 15, 2013 and October 25, 2013.

2010 Mathematics Subject Classification. Primary 11R39, 20G05, 22E50.
} 
$\boldsymbol{T}(k)_{0}$ of $\boldsymbol{T}(k)$. In Theorem 1, we calculate the image, under the local Langlands correspondence for tori, of all such characters which are trivial on $\boldsymbol{T}(k)_{0}$ and show that it is precisely inflation of the cocycles in $\mathrm{H}^{1}\left(W_{k} / I_{k}, \hat{\boldsymbol{T}}^{I_{k}}\right)$. In Theorem 2, we show that the orbits in $\mathrm{H}^{1}\left(W_{k} / I_{k}, \hat{\boldsymbol{T}}^{I_{k}}\right)$ of the relative Weyl group are in bijection with the semisimple conjugacy classes in $\hat{\boldsymbol{G}}^{I} \rtimes\left(W_{k} / I_{k}\right)$.

In [10, Chapter 11], a character of $\boldsymbol{T}(k)$ is called elementary if under the Langlands reciprocity map, it corresponds to a cocycle in $\mathrm{H}^{1}\left(W_{k}, \hat{\boldsymbol{T}}\right)$ which is the inflation of a cocyle in $\mathrm{H}^{1}\left(W_{k} / I_{k}, \hat{\boldsymbol{T}}^{I_{k}}\right)$. The question of characterizing the elementary characters is a natural one and some partial results are presented in 10, Chapter 11]. Our first theorem answers this question by showing that a character is elementary if and only if it is trivial on the Iwahori subgroup $\boldsymbol{T}(k)_{0}$.

The referee has pointed out that Lemma 5$]$ for arbitrary tori was independently observed in a recent work by Haines [3, Sec. 3.3.1]. In that article, Haines mentions that the result is implied by a more general result of Kaletha [5, Prop. 4.5.2]. A characteristic zero assumption is made in that result of Kaletha.

\section{Notation}

Let $k$ be a non-archimedian local field. Let $\boldsymbol{G}$ be a connected reductive group defined by $k$ which is quasi-split and split over a tamely ramified extension. We denote $\boldsymbol{G}(k)$ by $G$ and likewise for other algebraic groups. Let $K$ be a special maximal parahoric subgroup of $G$ corresponding to a special vertex $\nu$ in the BruhatTits building $\mathcal{B}\left(G_{\mathrm{ad}}\right)$. Let $\boldsymbol{A}$ denote a maximal split $k$-torus whose corresponding apartment in $\mathcal{B}\left(G_{\text {ad }}\right)$ contains $\nu$. Let $\boldsymbol{T}=Z_{\boldsymbol{G}} \boldsymbol{A}$, the centralizer of $\boldsymbol{A}$ in $\boldsymbol{T}$. Then $\boldsymbol{T}$ is a maximal torus in $\boldsymbol{G}$ since $\boldsymbol{G}$ is quasi-split. Let $W$ denote the relative Weyl group of $\boldsymbol{G}$. Let $\mathscr{H}(G, K)$ be the Hecke algebra of $K$-bi-invariant compactly supported complex-valued functions on $G$. Let $T_{c}$ and $T_{0}$ denote respectively the maximal compact subgroup and the Iwahori subgroup of $T$. Let $\hat{\boldsymbol{G}}$ denote the complex dual of $\boldsymbol{G}$ and $\hat{\boldsymbol{G}}_{\mathrm{ss}}$ the set of semisimple elements in $\hat{\boldsymbol{G}}$. Let $\operatorname{Inn}(\hat{\boldsymbol{G}})$ be the group of inner automorphisms of $\hat{\boldsymbol{G}}$. Let $\sigma=\sigma_{k}$ denote the Frobenius element in $W_{k} / I_{k}$, where $W_{k}$ is the Weil group of $k$ and $I=I_{k}$ is its inertia subgroup. We denote the identity component of an algebraic group $\mathscr{G}$ by $\mathscr{G}^{\circ}$.

\section{Statement of the theorems}

Theorem 1. A character is elementary if and only if it is trivial on the Iwahori subgroup. In other words, we have a commutative diagram:

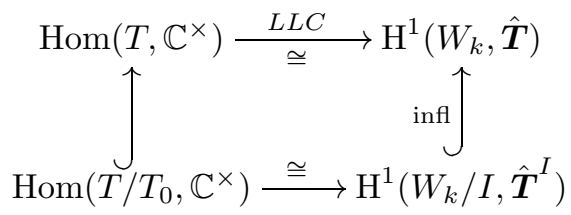

where LLC is the local Langlands correspondence for tori and infl is the inflation homomorphism.

Let $\operatorname{Rep}(G)$ denote the set of equivalent classes of smooth, irreducible admissible representations of $G$. 
Theorem 2. The $K$-spherical representations are in a natural bijection with the semisimple conjugacy classes in $\hat{\boldsymbol{G}}^{I} \rtimes \sigma$ via the local Langlands correspondence for tori. More precisely,

$$
\begin{aligned}
\left\{\pi \mid \pi \in \operatorname{Rep}(G), \pi^{K} \neq 0\right\} & \leftrightarrow \operatorname{Hom}_{\mathbb{C}}(\mathscr{H}(G, K), \mathbb{C}) \\
& \cong \operatorname{Hom}\left(T / T_{0}, \mathbb{C}^{\times}\right) / W \\
& \cong\left(\hat{\boldsymbol{T}}^{I}\right)_{\sigma} / W \\
& \cong\left(\hat{\boldsymbol{G}}^{I} \rtimes \sigma\right)_{\mathrm{ss}} / \operatorname{Inn}\left(\hat{\boldsymbol{G}}^{I}\right) .
\end{aligned}
$$

\section{LANGLANDS CORRESPONDENCE FOR TORI}

The following treatment of local Langlands correspondence for tori can be found in 11 .

3.1. The special case of an induced torus. Let $\boldsymbol{T}=R_{k^{\prime} / k} \mathbb{G}_{m}$ be an induced torus, when $k^{\prime}$ is a finite separable extension of $k$. Then $T=k^{\prime \times}$ and the group of characters $X^{*}(\boldsymbol{T})$ is canonically a free $\mathbb{Z}$-module with basis $W_{k} / W_{k^{\prime}}$. From this, it follows that $\hat{\boldsymbol{T}}$ is canonically isomorphic to $\operatorname{ind}_{W_{k^{\prime}}}^{W_{k}} \mathbb{C}^{\times}$. We get

$$
\begin{aligned}
\operatorname{Hom}\left(T, \mathbb{C}^{\times}\right) & \cong \operatorname{Hom}\left(k^{\prime \times}, \mathbb{C}^{\times}\right) \\
& \cong \operatorname{Hom}\left(W_{k^{\prime}}, \mathbb{C}^{\times}\right) \\
& \cong \mathrm{H}^{1}\left(W_{k^{\prime}}, \mathbb{C}^{\times}\right) \\
& \cong \mathrm{H}^{1}\left(W_{k}, \operatorname{ind}_{W_{k^{\prime}}}^{W_{k}} \mathbb{C}^{\times}\right) \\
& \cong \mathrm{H}^{1}\left(W_{k}, \hat{\boldsymbol{T}}\right) .
\end{aligned}
$$

The isomorphism (3.1) follows by class field theory and the isomorphism (3.2) by Shapiro's lemma.

\subsection{The LLC for tori in general.}

Theorem ([11, 7.5 Theorem]). There is a unique family of homomorphisms

$$
\varphi_{\boldsymbol{T}}: \operatorname{Hom}\left(T, \mathbb{C}^{\times}\right) \rightarrow \mathrm{H}^{1}\left(W_{k}, \hat{\boldsymbol{T}}\right)
$$

with the following properties:

(1) $\varphi_{\boldsymbol{T}}$ is additive functorial in $\boldsymbol{T}$; i.e., it is a morphism between two additive functors from the category of tori over $k$ to the category of abelian groups.

(2) For $\boldsymbol{T}=R_{k^{\prime} / k} \mathbb{G}_{m}$, where $k^{\prime} / k$ is a finite separable extension, $\varphi_{\boldsymbol{T}}$ is the isomorphism described in Section 3.1 .

\section{Proof of Theorem 1}

Lemma 3. Let $\boldsymbol{T}$ be a torus defined over $k$. Then there exists an isomorphism

$$
\kappa_{\boldsymbol{T}}: \operatorname{Hom}\left(T / T_{0}, \mathbb{C}^{\times}\right) \rightarrow \mathrm{H}^{1}\left(W_{k} / I, \hat{\boldsymbol{T}}^{I}\right) .
$$

Moreover, the isomorphism $k_{\boldsymbol{T}}$ is additive functorial in $\boldsymbol{T}$.

Proof. We have by the Kottwitz isomorphism [7, Sec. 7] (see also [4, Prop. 1.0.2])

$$
\begin{aligned}
T / T_{0} & \cong\left(\left(X^{*}(\hat{\boldsymbol{T}})\right)_{I}\right)^{\sigma} \\
& \cong X^{*}\left(\left(\hat{\boldsymbol{T}}^{I}\right)_{\sigma}\right) .
\end{aligned}
$$


Therefore,

$$
\begin{aligned}
\operatorname{Hom}\left(T / T_{0}, \mathbb{C}^{\times}\right) & \cong \operatorname{Hom}\left(X^{*}\left(\left(\hat{\boldsymbol{T}}^{I}\right)_{\sigma}\right), \mathbb{C}^{\times}\right) \\
& \cong\left(\hat{\boldsymbol{T}}^{I}\right)_{\sigma} \\
& \cong \mathrm{H}^{1}\left(W_{k} / I, \hat{\boldsymbol{T}}^{I}\right) .
\end{aligned}
$$

The isomorphism in equation (4.2) is by Cartier duality. The functoriality of $\kappa_{T}$ follows from the functoriality of the Kottwitz isomorphism.

Remark 4. From the relation

$$
T / T_{c} \cong\left(X^{*}(\hat{\boldsymbol{T}})_{I}\right)^{\sigma} / \text { torsion, }
$$

one similarly obtains the isomorphism

$$
\operatorname{Hom}\left(T / T_{c}, \mathbb{C}^{\times}\right) \cong \mathrm{H}^{1}\left(W_{k} / I,\left(\hat{\boldsymbol{T}}^{I}\right)^{\circ}\right) .
$$

Lemma 5. Let $k^{\prime} / k$ be a finite separable extension and put $\boldsymbol{T}=R_{k^{\prime} / k}\left(\mathbb{G}_{m}\right)$. Then the isomorphism $\operatorname{Hom}\left(T / T_{0}, \mathbb{C}^{\times}\right) \cong \mathrm{H}^{1}\left(W_{k} / I, \hat{\boldsymbol{T}}^{I}\right)$ obtained from the Kottwitz isomorphism in Lemma 3 is the same as the one induced from the local Langlands correspondence for tori.

Proof. Since $\boldsymbol{T}$ is an induced torus, $X^{*}(\boldsymbol{T})$ is canonically a free $\mathbb{Z}$-module with basis $W_{k} / W_{k^{\prime}}$. Consequently, $\hat{\boldsymbol{T}}$ is simply $\operatorname{ind}_{W_{k^{\prime}}}^{W_{k}} \mathbb{C}^{\times}$. Let $\mathscr{O}$ and $\mathscr{O}^{\prime}$ be the ring of integers in $k$ and $k^{\prime}$ respectively. We have

$$
\begin{aligned}
\mathrm{H}^{1}\left(W_{k} / I_{k}, \hat{\boldsymbol{T}}^{I_{k}}\right) & \cong\left(\hat{\boldsymbol{T}}^{I_{k}}\right)_{\sigma_{k}} \\
& \cong\left(\left(\operatorname{ind}_{W_{k}}^{W_{k}} \mathbb{C}^{\times}\right)^{I_{k}}\right)_{\sigma_{k}} \\
& \cong \mathrm{H}^{1}\left(W_{k} / I_{k},\left(\operatorname{ind}_{W_{k} W^{\prime}}^{W^{\times}}\right)^{I_{k}}\right) \\
& \cong \mathrm{H}^{1}\left(W_{k^{\prime}} / I_{k^{\prime}}, \mathbb{C}^{\times}\right) \\
& \cong \operatorname{Hom}\left(k^{\prime \times} / \mathscr{O}^{\prime \times}, \mathbb{C}^{\times}\right) \\
& \cong \operatorname{Hom}\left(T / T_{0}, \mathbb{C}^{\times}\right) .
\end{aligned}
$$

Here, the isomorphism in (4.4) is induced by the isomorphism in Shapiro's lemma:

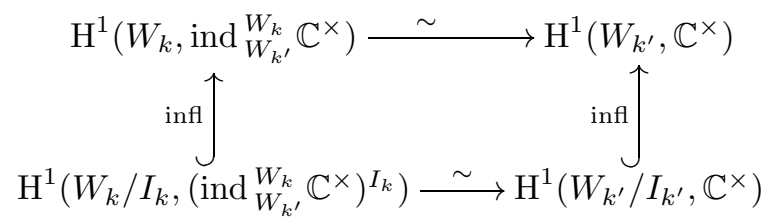

Thus the local Langlands correspondence also induces an isomorphism:

$$
\varphi_{\boldsymbol{T}}: \operatorname{Hom}\left(T / T_{0}, \mathbb{C}^{\times}\right) \cong \mathrm{H}^{1}\left(W_{k} / I, \hat{\boldsymbol{T}}^{I}\right) .
$$

The Kottwitz isomorphism for induced tori is constructed as follows (see 7 , Sec. 7.2]): the homomorphism

$$
v_{T}^{\prime}: T \rightarrow \operatorname{Hom}\left(X^{*}(\boldsymbol{T}), \mathbb{Z}\right),
$$

sending $t \in T$ to the homomorphism

$$
\lambda \mapsto \operatorname{ord}(\lambda(t)),
$$


induces an isomorphism

$$
v_{T}: T / T_{0} \cong \operatorname{Hom}\left(X^{*}(\boldsymbol{T})^{I}, \mathbb{Z}\right)^{\sigma} .
$$

Also, the homomorphism

$$
q_{T}^{\prime}: X_{*}(\boldsymbol{T}) \rightarrow \operatorname{Hom}\left(X^{*}(\boldsymbol{T}), \mathbb{Z}\right),
$$

which sends $\mu \in X_{*}(T)$ to the homomorphism

$$
\lambda \mapsto\langle\lambda, \mu\rangle,
$$

induces an isomorphism

$$
q_{T}:\left(X_{*}(\boldsymbol{T})_{I}\right)^{\sigma} \cong \operatorname{Hom}\left(X^{*}(\boldsymbol{T})^{I}, \mathbb{Z}\right)^{\sigma} .
$$

From the equations (4.6) and (4.7), we get the Kottwitz isomorphism for the induced torus $\boldsymbol{T}$ :

$$
w_{T}: T / T_{0} \cong X^{*}\left(\left(\hat{\boldsymbol{T}}^{I}\right)_{\sigma}\right),
$$

where we used the identifications $\left(X_{*}(\boldsymbol{T})_{I}\right)^{\sigma}=\left(X^{*}(\hat{\boldsymbol{T}})_{I}\right)^{\sigma} \cong X^{*}\left(\left(\hat{\boldsymbol{T}}^{I}\right)_{\sigma}\right)$. This map $w_{T}$ induces the map $\kappa_{\boldsymbol{T}}: \operatorname{Hom}\left(T / T_{0}, \mathbb{C}^{\times}\right) \cong \mathrm{H}^{1}\left(W_{k} / I, \hat{\boldsymbol{T}}^{I}\right)$ in Lemma 3 .

We identify $T / T_{0}$ with $\mathbb{Z}$ by the isomorphisms

$$
T / T_{0} \cong k^{\prime \times} / \mathscr{O}^{\prime \times} \cong \varpi^{\prime \mathbb{Z}} \cong \mathbb{Z}
$$

Here $\varpi^{\prime}$ is the uniformizer in $k^{\prime}$. Let $J$ be a $W_{k}$-stable basis of $X^{*}(\boldsymbol{T})$. Choose any $\lambda \in J$ and let $J_{\lambda} \subset J$ be the orbit of $\lambda$ in $J$ under the action of $I_{k}$. Let $\chi=\sum_{\mu \in J_{\lambda}} \mu$. Then $\chi \in X^{*}(\hat{\boldsymbol{T}})^{I_{k}}$.

Let $f \in \operatorname{Hom}\left(T / T_{0}, \mathbb{C}^{\times}\right)$and let $c=f(1)$ (under the identification in equation (4.8)). Then both $\kappa_{\boldsymbol{T}}$ and $\varphi_{\boldsymbol{T}}$ map $f$ to the cocycle $\phi_{f} \in \mathrm{H}^{1}\left(W_{k} / I, \hat{\boldsymbol{T}}^{I}\right)$ defined by $\sigma \mapsto \chi \otimes c$. Thus $\kappa_{\boldsymbol{T}}=\varphi_{\boldsymbol{T}}$. This completes the proof of the lemma.

Proposition 6. There is a unique family of homomorphisms,

$$
\varphi_{\boldsymbol{T}}: \operatorname{Hom}\left(T / T_{0}, \mathbb{C}^{\times}\right) \rightarrow \mathrm{H}^{1}\left(W_{k}, \hat{\boldsymbol{T}}\right),
$$

with the following properties:

(1) $\varphi_{\boldsymbol{T}}$ is additive functorial in $\boldsymbol{T}$; i.e., it is a morphism between two additive functors from the category of tori over $k$ to the category of abelian groups.

(2) For $\boldsymbol{T}=R_{k^{\prime} / k} \mathbb{G}_{m}$, where $k^{\prime} / k$ is a finite Galois extension, $\varphi_{\boldsymbol{T}}$ is the homomorphism induced from the local Langlands correspondence for tori.

Proof. Since the isomorphism $\kappa_{\boldsymbol{T}}$ in Lemma 3 is additive functorial in $\boldsymbol{T}$, we thus get an additive functorial family of homomorphisms

$$
\varphi_{\boldsymbol{T}}: \operatorname{Hom}\left(T / T_{0}, \mathbb{C}^{\times}\right) \longrightarrow \mathrm{H}^{1}\left(W_{k} / I, \hat{\boldsymbol{T}}^{I}\right) \stackrel{\text { infl }}{\longrightarrow} \mathrm{H}^{1}\left(W_{k}, \hat{\boldsymbol{T}}\right) .
$$

This shows existence. To show uniqueness, let $\boldsymbol{T}$ be a given torus defined over $k$ and let $k^{\prime} / k$ be a finite Galois extension such that $\boldsymbol{T}$ is split over $k^{\prime}$. Let $\boldsymbol{T}^{\prime}=$ $R_{k^{\prime} / k}\left(\boldsymbol{T} \otimes_{k} k^{\prime}\right)$. Then $\boldsymbol{T}^{\prime}$ is isomorphic to a direct sum of $d=\operatorname{dim}(\boldsymbol{T})$ tori of 
the form $R_{k^{\prime} / k}\left(\mathbb{G}_{m}\right)$ and there is a natural embedding $\boldsymbol{T} \hookrightarrow \boldsymbol{T}^{\prime}$. This gives an embedding $T / T_{0} \hookrightarrow T^{\prime} / T_{0}^{\prime}$. By (1), there is a commutative diagram

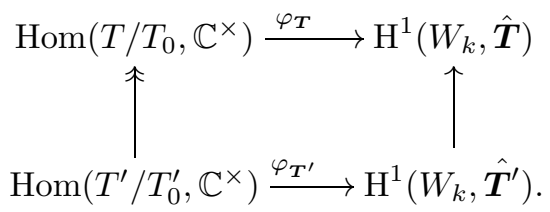

Notice that $\varphi_{\boldsymbol{T}^{\prime}}$ is completely determined by (2). Now given $x \in \operatorname{Hom}\left(T / T_{0}, \mathbb{C}^{\times}\right)$, we can lift it to $x^{\prime} \in \operatorname{Hom}\left(T^{\prime} / T_{0}^{\prime}, \mathbb{C}^{\times}\right)$. It follows that $\varphi_{\boldsymbol{T}}(x)$ is the image of $\varphi_{\boldsymbol{T}^{\prime}}\left(x^{\prime}\right)$ under the vertical arrow on the right and is hence determined by (1) and (2).

Theorem 7. Let $\boldsymbol{T}$ be a torus defined over $k$. Then the local Langlands correspondence for tori induces the isomorphism

$$
\operatorname{Hom}\left(T / T_{0}, \mathbb{C}^{\times}\right) \cong \mathrm{H}^{1}\left(W_{k} / I, \hat{\boldsymbol{T}}^{I}\right) .
$$

Proof. By the above proposition, it follows that the homomorphism $\operatorname{Hom}\left(T / T_{0}, \mathbb{C}^{\times}\right)$ $\rightarrow \mathrm{H}^{1}\left(W_{k}, \hat{\boldsymbol{T}}\right)$, determined by the Kottwitz map, must be the same as the one determined by LLC. Therefore, the image of $\operatorname{Hom}\left(T / T_{0}, \mathbb{C}^{\times}\right)$under LLC must be the same as the one determined by $\kappa_{\boldsymbol{T}}$, which is $\mathrm{H}^{1}\left(W_{k} / I, \hat{\boldsymbol{T}}^{I}\right)$. This proves the theorem.

\section{Some ReSults ABOUt InERTia FiXed Groups}

Let $\boldsymbol{B}$ be a Borel subgroup of $\boldsymbol{G}$ containing the maximal torus $\boldsymbol{T}$ of $\boldsymbol{G}$. The triple $(\boldsymbol{G}, \boldsymbol{B}, \boldsymbol{T})$ determines a based root datum. Let $(\hat{\boldsymbol{G}}, \hat{\boldsymbol{B}}, \hat{\boldsymbol{T}})$ be the triple defined over $\mathbb{C}$ which corresponds to the dual based root datum. Since $\boldsymbol{G}$ is tamely ramified, the inertia group acts on $(\hat{\boldsymbol{G}}, \hat{\boldsymbol{B}}, \hat{\boldsymbol{T}})$ via a cyclic group $(\tau)$. Let $\boldsymbol{W}$ be the Weyl group of $\hat{\boldsymbol{G}}$. Let $\hat{\boldsymbol{T}}_{\text {ad }}$ be the image of $\hat{\boldsymbol{T}}$ in $\hat{\boldsymbol{G}}_{\text {ad }}$, the adjoint group of $\hat{\boldsymbol{G}}$. Let $\hat{\boldsymbol{Z}}$ be the center of $\hat{\boldsymbol{G}}$.

The following results are given in [8, Sec. 1.1 (Theorem 1.1.A and the paragraphs following it)].

Theorem 8 (Kottwitz-Shelstad).

(1) $\left(\hat{\boldsymbol{G}}^{\tau}\right)^{\circ}$ is a connected reductive group.

(2) $\left(\hat{\boldsymbol{B}}^{\tau}\right)^{\circ}$ is a Borel subgroup of $\left(\hat{\boldsymbol{G}}^{\tau}\right)^{\circ}$ containing its maximal torus $\left(\hat{\boldsymbol{T}}^{\tau}\right)^{\circ}$.

(3) $\boldsymbol{W}^{\tau}$ is the Weyl group of $\left(\hat{\boldsymbol{G}}^{\tau}\right)^{\circ}$.

(4) $\hat{\boldsymbol{G}}^{\tau}=\hat{\boldsymbol{Z}}^{\tau}\left(\hat{\boldsymbol{G}}^{\tau}\right)^{\circ}$.

(5) $\left(\hat{\boldsymbol{T}}_{\mathrm{ad}}\right)^{\tau}$ is connected.

From (5), we immediately obtain

$\left(5^{\prime}\right) \hat{\boldsymbol{T}}^{\tau}=\hat{\boldsymbol{Z}}^{\tau}\left(\hat{\boldsymbol{T}}^{\tau}\right)^{\circ}$.

Remark 9. As pointed out by the referee, these facts can be proved as follows. The fact that $\left(\hat{\boldsymbol{T}}_{\mathrm{ad}}\right)^{\tau}$ is connected follows from [9, remark at the end of Cor. 9.12]. Using this and [12, Lemma 4.6], it follows that $\left(\hat{\boldsymbol{G}}_{\mathrm{ad}}\right)^{\tau}$ is connected. From this, (4) follows. The fact that $\left(\hat{\boldsymbol{G}}^{\tau}\right)^{\circ}$ is reductive follows from a general lemma of Kottwitz [6, 10.1.2]. The fact that $\boldsymbol{W}^{\tau}$ is the Weyl group of $\left(\hat{\boldsymbol{G}}^{\tau}\right)^{\circ}$ can be proved by adapting the proof of Theorem 8.2 of [9]. Since $\tau$ fixes a splitting, this allows one to take $t=1$ in that proof. 


\section{Proof of Theorem 2}

Lemma 10. $\operatorname{Hom}_{\mathbb{C}}(\mathscr{H}(G, K), \mathbb{C}) \cong \operatorname{Hom}\left(T / T_{0}, \mathbb{C}^{\times}\right) / W$.

Proof. $\mathscr{H}(G, K) \cong \mathbb{C}\left[T / T_{0}\right]^{W}$ by [4, Theorem 1.0.1]. Therefore

$$
\begin{aligned}
\operatorname{Hom}_{\mathbb{C}}(\mathscr{H}(G, K), \mathbb{C}) & \cong \operatorname{Hom}_{\mathbb{C}}\left(\mathbb{C}\left[T / T_{0}\right]^{W}, \mathbb{C}\right) \\
& \cong \operatorname{Hom}_{\mathbb{C}}\left(\mathbb{C}\left[T / T_{0}\right], \mathbb{C}\right) / W \\
& \cong \operatorname{Hom}\left(T / T_{0}, \mathbb{C}^{\times}\right) / W .
\end{aligned}
$$

\section{Proposition 11.}

$$
\left(\hat{\boldsymbol{T}}^{I}\right)_{\sigma} / W \cong Z(\hat{\boldsymbol{G}})^{I}\left(\hat{\boldsymbol{G}}^{I}\right)_{\mathrm{ss}}^{\circ} \rtimes \sigma / \operatorname{Inn}\left(Z(\hat{\boldsymbol{G}})^{I}\left(\hat{\boldsymbol{G}}^{I}\right)^{\circ}\right) .
$$

Proof. Let $\boldsymbol{W}$ be, as before, the Weyl group of $\hat{\boldsymbol{G}}$. By Theorem $8(3), \boldsymbol{W}^{\tau}$ is the Weyl group of $\left(\hat{\boldsymbol{G}}^{\tau}\right)^{\circ}$. Since $\left(\hat{\boldsymbol{G}}^{\tau}\right)^{\circ}$ is reductive (by Theorem $8(1)$ ), it follows from the proof of [1, Lemma 6.5] that we have a surjection

$$
\left(\hat{\boldsymbol{T}}^{\tau}\right)^{\circ} \rightarrow\left(\left(\hat{\boldsymbol{G}}^{\tau}\right)^{\circ} \rtimes \sigma\right)_{\mathrm{ss}} / \operatorname{Inn}\left(\left(\hat{\boldsymbol{G}}^{\tau}\right)^{\circ}\right) .
$$

By Theorem $8\left(5^{\prime}\right)$, this implies

$$
\hat{\boldsymbol{T}}^{\tau} \rightarrow\left(\hat{\boldsymbol{Z}}^{\tau}\left(\hat{\boldsymbol{G}}^{\tau}\right)^{\circ} \rtimes \sigma\right)_{\mathrm{ss}} / \operatorname{Inn}\left(\hat{\boldsymbol{Z}}^{\tau}\left(\hat{\boldsymbol{G}}^{\tau}\right)^{\circ}\right) .
$$

Denote the $\sigma$-action on an element $g \in \hat{\boldsymbol{G}}$ by $g^{\sigma}$. Let $s, t \in \hat{\boldsymbol{T}}^{\tau}$ be such that there exists $g \in \hat{\boldsymbol{Z}}^{\tau}\left(\hat{\boldsymbol{G}}^{\tau}\right)^{\circ}$ satisfying $g^{-1} s g^{\sigma}=t$. As defined in Section [5] let $\hat{\boldsymbol{B}}$ be the Borel subgroup of $\hat{\boldsymbol{G}}$ containing the maximal torus $\hat{\boldsymbol{T}}$. Write $g=u n v$ using Bruhat decomposition, where $u, v$ are in the unipotent radical of $\hat{\boldsymbol{B}}$ and $n$ is in the normalizer $\hat{\boldsymbol{N}}$ of $\hat{\boldsymbol{T}}$. Also, $g=z g_{0}$ for some $z \in \hat{\boldsymbol{Z}}^{\tau}$ and $g_{0} \in\left(\hat{\boldsymbol{G}}^{\tau}\right)^{\circ}$. Let $g_{0}=u_{0} n_{0} v_{0}$ be the Bruhat decomposition of $g_{0}$ in $\left(\hat{\boldsymbol{G}}^{\tau}\right)^{\circ}$ with respect to the Borel $\left(\hat{\boldsymbol{B}}^{\tau}\right)^{\circ}$ and maximal torus $\left(\hat{\boldsymbol{T}}^{\tau}\right)^{\circ}$. Then $u=u_{0}, v=v_{0}$ and $n=z n_{0}$. Thus,

$$
\begin{aligned}
g^{-1} s g^{\sigma}=t & \Longrightarrow s u^{\sigma} n^{\sigma} v^{\sigma}=u n v t \\
& \Longrightarrow s u^{\sigma} s^{-1} s n^{\sigma} v^{\sigma}=u n t t^{-1} v t \\
& \Longrightarrow s n^{\sigma}=n t \\
& \Longrightarrow s z^{\sigma} n_{0}^{\sigma}=z n_{0} t .
\end{aligned}
$$

Let $\bar{n}_{0}$ denote the image of $n_{0}$ in $\boldsymbol{W}$. Then $n_{0}^{\tau}=n_{0} \Longrightarrow \bar{n}_{0} \in \boldsymbol{W}^{\tau}$. Also (6.3) implies $\bar{n}_{0}^{\sigma}=\bar{n}_{0}$. Thus $\bar{n}_{0} \in \boldsymbol{W}^{\tau, \sigma}$. Using again the fact that $\left(\hat{\boldsymbol{G}}^{\tau}\right)^{\circ}$ is reductive, it follows from the proof of Lemma 6.2 in [1 that there exists $p \in$ $N_{\left(\hat{\boldsymbol{G}}^{\tau}\right)^{\circ}}\left(\hat{\boldsymbol{T}}^{\tau}\right)^{\circ}$ such that $p^{\sigma}=p$ and $n_{0} \in p\left(\hat{\boldsymbol{T}}^{\tau}\right)^{\circ}$. This fact is also shown in the proof of [12, Lemma 4.7]. Let $n_{0}=p q$ for some $q \in\left(\hat{\boldsymbol{T}}^{\tau}\right)^{\circ}$. Then,

$$
z^{-1} z^{\sigma} q^{-1} p^{-1} s p^{\sigma} q^{\sigma}=t .
$$

Let $r=z q \in \hat{\boldsymbol{T}}^{\tau}$. Then $r^{-1} p^{-1} s p r^{\sigma}=t$. Thus $\bar{s}=\bar{t}$ where $\bar{s}$ and $\bar{t}$ represent the class of $s$ and $t$ in $\hat{\boldsymbol{T}}_{\sigma}^{\tau} / \boldsymbol{W}^{\tau, \sigma}=\left(\hat{\boldsymbol{T}}^{I}\right)_{\sigma} / W$.

Using Theorem 8(4), we obtain

Corollary 12. $\left(\hat{\boldsymbol{T}}^{I}\right)_{\sigma} / W \cong\left(\hat{\boldsymbol{G}}^{I} \rtimes \sigma\right)_{\mathrm{ss}} / \operatorname{Inn}\left(\hat{\boldsymbol{G}}^{I}\right)$. 
Write $\boldsymbol{B}=\boldsymbol{T} \boldsymbol{U}$, where $\boldsymbol{B}$ is as before, the Borel subgroup of $\boldsymbol{G}$ containing $\boldsymbol{T}$, and where $\boldsymbol{U}$ is the unipotent radical of $\boldsymbol{B}$. Using the Iwasawa decomposition [4, Corr. 9.1.2], we can define the spherical functions

$$
\Phi_{K, \chi}(t u k)=\chi(m) \delta^{1 / 2}(m),
$$

as in [2], where $t \in T, u \in U, k \in K$ and $\delta$ is the modulus function. The proof of the bijection

$$
\left\{\pi \mid \pi \in \operatorname{Rep}(G), \pi^{K} \neq 0\right\} \leftrightarrow \operatorname{Hom}_{\mathbb{C}}(\mathscr{H}(\boldsymbol{G}, K), \mathbb{C})
$$

is then identical to the case when $\boldsymbol{G}$ is unramified and $K$ is hyperspecial, which is given in 2]. This completes the proof of Theorem 2 .

\section{ACKNOWLEDGEMENTS}

The author would like to thank his advisor Jiu-Kang Yu for his suggestion of this problem and for his careful mentoring throughout. He would also like to thank Sungmun Cho for many helpful suggestions. He is thankful to Sandeep Varma for pointing him to the reference for Theorem 8 and for his careful proofreading.

The author is very thankful to the referee for many helpful inputs, particularly in the proof of Proposition [1]. The referee pointed out the fact that $\hat{\boldsymbol{Z}}^{I}\left(\hat{\boldsymbol{G}}^{I}\right)^{\circ}$ is simply $\hat{\boldsymbol{G}}^{I}$ and also provided a proof of this fact. This makes the statement of Proposition 11 appear more natural.

\section{REFERENCES}

[1] A. Borel, Automorphic L-functions, Automorphic forms, representations and $L$-functions (Proc. Sympos. Pure Math., Oregon State Univ., Corvallis, Ore., 1977), Part 2, Proc. Sympos. Pure Math., XXXIII, Amer. Math. Soc., Providence, R.I., 1979, pp. 27-61. MR.546608 (81m:10056)

[2] P. Cartier, Representations of p-adic groups: a survey, Automorphic forms, representations and $L$-functions (Proc. Sympos. Pure Math., Oregon State Univ., Corvallis, Ore., 1977), Part 1, Proc. Sympos. Pure Math., XXXIII, Amer. Math. Soc., Providence, R.I., 1979, pp. 111-155. MR.546593 (81e:22029)

[3] T. Haines, The stable Bernstein center and test functions for Shimura varieties, arXiv:1304.6293. To appear in the proceedings for the London Mathematical Society - EPSRC Durham Symposium on Automorphic Forms and Galois Representations, Durham, July $18-28,2011$.

[4] Thomas J. Haines and Sean Rostami, The Satake isomorphism for special maximal parahoric Hecke algebras, Represent. Theory 14 (2010), 264-284, DOI 10.1090/S1088-4165-10-00370-5. MR:2602034(2011g:20077)

[5] T. Kaletha, Epelagic L-packets and rectifying characters, arXiv:1209.1720

[6] Robert E. Kottwitz, Stable trace formula: cuspidal tempered terms, Duke Math. J. 51 (1984), no. 3, 611-650, DOI 10.1215/S0012-7094-84-05129-9. MR757954 (85m:11080)

[7] Robert E. Kottwitz, Isocrystals with additional structure. II, Compositio Math. 109 (1997), no. 3, 255-339, DOI 10.1023/A:1000102604688. MR1485921(99e:20061)

[8] Robert E. Kottwitz and Diana Shelstad, Foundations of twisted endoscopy (English, with English and French summaries), Astérisque 255 (1999), vi+190. MR1687096 (2000k:22024)

[9] Robert Steinberg, Endomorphisms of linear algebraic groups, Memoirs of the American Mathematical Society, No. 80, American Mathematical Society, Providence, R.I., 1968. MR.0230728 (37 \#6288)

[10] Rainer Weissauer, Endoscopy for GSp(4) and the cohomology of Siegel modular threefolds, Lecture Notes in Mathematics, vol. 1968, Springer-Verlag, Berlin, 2009. MR2498783 (2010h:11086) 
[11] Jiu-Kang Yu, On the local Langlands correspondence for tori, Ottawa lectures on admissible representations of reductive $p$-adic groups, Fields Inst. Monogr., vol. 26, Amer. Math. Soc., Providence, RI, 2009, pp. 177-183. MR2508725 (2009m:11201)

[12] Xinwen Zhu, The geometric Satake correspondence for ramified groups, arXiv:1107.5762

Einstein Institute of Mathematics, The Hebrew University of Jerusalem, Jerusalem, 91904, ISRAEL

E-mail address: mmishra@math.huji.ac.il 